\title{
Bronchial thermoplasty: activations predict response
}

\author{
David Langton ${ }^{1,2^{*}}$, Joy Sha ${ }^{1}$, Alvin Ing $^{3}$, David Fielding ${ }^{4}$, Francis Thien ${ }^{5}$ and Virginia Plummer ${ }^{2}$
}

\begin{abstract}
Background: Bronchial thermoplasty (BT) is an emerging bronchoscopic intervention for the treatment of severe asthma. The predictive factors for clinical response to BT are unknown. We examined the relationship between the number of radiofrequency activations applied and the treatment response observed.

Methods: Data were collected from 24 consecutive cases treated at three Australian centres from June 2014 to March 2016. The baseline characteristics were collated along with the activations delivered. The primary response measure was change in the Asthma Control Questionnaire-5 (ACQ-5) score measured at 6 months post BT. The relationship between change in outcome parameters and the number of activations delivered was explored.

Results: All patients met the ERS/ATS definition for severe asthma. At 6 months post treatment, mean ACQ- 5 improved from $3.3 \pm 1.1$ to $1.5 \pm 1.1, p<0.001$. The minimal clinically significant improvement in ACQ-5 of $\geq 0.5$ was observed in 21 out of 24 patients. The only significant variable that differed between the 21 responders and the three non-responders was the number of activations delivered, with $139 \pm 11$ activations in the non-responders, compared to $221 \pm 45$ activations in the responders $(p<0.01)$. A significant inverse correlation was found between change in ACQ-5 score and the number of activations, $r=-0.43(p<0.05)$.
\end{abstract}

Conclusions: The number of activations delivered during BT has a role in determining clinical response to treatment.

Keywords: Severe asthma, Bronchial thermoplasty, Response predictors, Activations, Technique

\section{Background}

Whilst most asthmatic patients can be well controlled using currently available inhaler therapy, there remains a small percentage of severely symptomatic patients whose daily lives are limited by their chronic condition [1]. Given the high prevalence of asthma worldwide, numerically this represents many millions of people with an unmet need for effective therapy [2].

In randomized clinical trials, bronchial thermoplasty (BT) has been shown to be an effective and safe additional modality for the management of patients with poorly controlled asthma despite standard therapy [3-5]. Typically, selected patients have daily symptoms, and frequent exacerbations requiring corticosteroids, despite high dose inhaled corticosteroids and long acting bronchodilators.

\footnotetext{
* Correspondence: davidlangton@phcn.vic.gov.au

'Department of Thoracic Medicine, Frankston Hospital, 2 Hastings Road, Frankston, VIC 3199, Australia

${ }^{2}$ Faculty of Medicine, Nursing and Health Sciences, Monash University, Melbourne, VIC, Australia

Full list of author information is available at the end of the article
}

BT is performed during flexible bronchoscopy using a radiofrequency catheter to deliver thermal injury to airways between 3 and $10 \mathrm{~mm}$ in size [6]. Both animal and human studies have demonstrated that, as result, there is a reduction in airway smooth muscle mass in the areas treated, whilst the airway mucosa recovers undamaged [7-9]. Airway smooth muscle is typically hypertrophied in asthma, responsible for producing variable bronchoconstriction and hence wheezing [10]. Clinical studies have demonstrated that 12 months following treatment, significant improvements are achieved in patient symptom scores, reliever medication usage, and asthma exacerbations requiring prednisolone [3-5]. There is however very little data regarding the characteristics of those who fail to respond to $\mathrm{BT}$, nor the contributing reasons.

It is possible that there is operator dependent variation in the adequacy of the radiofrequency treatment delivered to the airways during BT. If so, this could be responsible for a lack of response in some participants. Currently 
there is no routine clinical method of measuring airway smooth muscle thickness. However, a surrogate marker for treatment effectiveness can be found in the number of actuations delivered by the radiofrequency system. This measure is routinely available at every case, and is the total of the number of 10-s radiofrequency heat charges delivered to the airway. Whilst recommendations have been provided on the number of actuations that constitute effective treatment [11], there is an absence of existing evidence reporting on the relationship between activations and clinical outcomes. It is tempting to speculate that a lower number of actuations may reflect less airway smooth muscle treated, but this may also depend on patient factors such as airway size. Similarly, we cannot conclude whether higher numbers of total activations results in better asthma control, and if there is any impact on immediate post-procedure recovery.

Therefore, in this study, we examine retrospectively in our case series whether there is any relationship between number of radiofrequency actuations delivered and the patient response observed, as measured by improvement in symptom scores.

\section{Methods}

\section{Study participants}

Twenty four consecutive participants with severe, poorly controlled asthma were treated with BT between June 2014 and March 2016 at three Australian university teaching hospitals. All patients had been under the regular care of a specialist respiratory physician prior to referral to the treating proceduralist. Patients were chosen for bronchial thermoplasty at the discretion of the treating team. Subsequently, the medical records of each patient were reviewed and the baseline characteristics of the participants collated, including age, gender, height, weight, preventative and reliever asthma medication, smoking history, spirometry, and the Asthma Control Questionnaire Score (ACQ-5).

\section{Procedure}

Each proceduralist was head of bronchoscopic services in their hospital and had at least 25 years experience in performing bronchoscopy. All proceduralists had been trained in using the Alair bronchial thermoplasty system (Alair, Boston Scientific, NSW, Australia), and used the Olympus BF Q190 (Olympus Australia, Victoria, Australia) bronchoscope under general anaesthesia. The cases reported were the first cases that the proceduralists had undertaken. BT was conducted in three treatments, 3 to 4 weeks apart, starting with the right lower lobe, then left lower lobe, and, at the last treatment, both upper lobes. Patients were treated with oral prednisolone for 3 days before and 3 days after the procedure. All patients were electively observed in hospital for $24-48 \mathrm{~h}$ post procedure, during which time they also received nebulised bronchodilators. The number of radiofrequency activations was recorded for each treatment session.

\section{Measurements}

The primary outcome measure chosen was change in the ACQ-5 from baseline. This measure is known to exhibit stability over time and to be responsive to improvements in asthma symptoms [12]. It was administered by study nurses immediately prior to commencing $\mathrm{BT}$, and again 6 months following the final bronchoscopy. These nurses were blinded to intraoperative care such as the number of activations administered. Spirometry was conducted in accredited respiratory laboratories by experienced scientific staff and according to European Respiratory Society/American Thoracic Society (ERS/ ATS) standards [13]. For uniformity between sites, the predicted equations used were from Quanjer [14].

\section{Safety}

An adverse event was recorded for any participant who required admission of longer than $48 \mathrm{~h}$, or any participant who was readmitted to hospital for any cause within 30 days of any procedure.

\section{Ethics}

Approval to collate and audit data as part of quality assurance was provided by the Human Research and Ethics Committee at each participating institution. Participants were assigned a unique study-specific identifier number, and clinical data was shared between institutions using these numbers so that no individually identifiable data was disclosed. Specific permission to use the ACQ-5 in this project was sought from, and granted by, the author Elizabeth Juniper. All participants provided informed consent to treatment and data collection.

\section{Statistical analysis}

SPSS version 24 (IBM corporation, New York, USA) was used for all statistical analyses. Grouped data has been reported as mean \pm standard deviation unless the data was not parametric, in which case median (Interquartile range(IQR)) was used. A paired t-test was undertaken to compare all paired sets of normally distributed data, whilst a Wilcoxon signed rank test was used for paired non-parametric data. Where the sample size was very small, the data was assumed to be non parametric and a Mann-Whitney U test was used. Statistical significance was taken as $p<0.05$ for a two-tailed test. Pearson's Correlation Coefficient was calculated for bivariate continuous normally distributed data, and univariate linear regression was performed if a significant relationship was observed. The mean radiofrequency treatment 
activations per patient at the three different participating institutions were compared by ANOVA. A multivariate linear regression model was created to examine clinical factors that might predict the number of activations delivered to an individual patient.

\section{Results}

\section{Baseline characteristics}

Twenty four patients, 16 females and 8 males, completed treatment and 6 months follow-up. The mean age was $55.4 \pm 12.6$ years (range: $27-75$ ). The mean BMI was $28.8 .8 \pm 6.7$ (range 22.2-46.9). 22 patients were neversmokers, whilst 2 were ex-smokers with a less than 10 pack-year history. The mean baseline prebronchodilator $\mathrm{FEV}_{1}$ was $61.8 \pm 15.9 \%$ predicted (range: $33-95 \%$ ); 12 cases $(50 \%)$ had an $\mathrm{FEV}_{1}$ of $<60 \%$ predicted, and six cases $\mathrm{FEV}_{1}<50 \%$ predicted. The mean change in baseline $\mathrm{FEV}_{1}$ after administration of salbutamol was $13.7 \pm 12.7 \%$ (range $0-46.7 \%$ ).

All participants had been prescribed high doses of inhaled corticosteroids, mean beclomethasone equivalent dose of $2095 \pm 450 \mathrm{mcg}$ daily (range: $1000-3000 \mathrm{mcg}$ ). Twelve patients $(50 \%)$ were taking maintenance oral prednisolone (median dose $10 \mathrm{mg} /$ day, range $4-20 \mathrm{mg}$ ). All patients $(100 \%)$ were taking long-acting beta ${ }_{2}$ agonists and long-acting muscarinic antagonists. Additional preventative therapy included leukotriene receptor antagonists (42\%), omalizumab (29\%), and methotrexate (17\%).

Every patient met the ERS/ATS definition for severe asthma, by fulfilling at least one of the four criteria [1]. Symptom control remained poor despite the extensive preventative treatment. The mean ACQ-5 score was $3.3 \pm$ 1.1. Patients used a median of eight salbutamol puffs per day for rescue reliever therapy (IQR 4-15) and, in the 6 months prior to treatment, there was a median of two exacerbations requiring prednisolone per patient (IQR 0-5).

\section{Response to treatment}

Table 1 presents the comparison at 6 months post treatment with baseline for the main outcome parameters. Favourable responses to bronchial thermoplasty were seen in all parameters including $\mathrm{FEV}_{1}$.

\section{Responder analysis}

An improvement in ACQ-5 of greater than 0.5 units (the minimal clinically significant difference) [12] was observed in 21 of 24 participants (88\%). The three nonresponders were compared with their counterparts across a range of clinical variables, including age, gender, baseline $\mathrm{FEV}_{1} \%$, baseline bronchodilator response, medication usage and exacerbation frequency (Table 2). The only significant difference between the two groups
Table 1 Response to BT treatment, $n=24$

\begin{tabular}{llll}
\hline & Baseline & 6 months post & $p$ \\
\hline ACQ-5 & $3.3 \pm 1.1$ & $1.5 \pm 1.1$ & $<0.001^{\mathrm{a}}$ \\
FEV 1 \%predicted & $61.8 \pm 15.9$ & $68.7 \pm 15.6$ & $<0.05^{\mathrm{a}}$ \\
Salbutamol puffs/day & $8(11)$ & $2(2)$ & $<0.001^{\mathrm{b}}$ \\
Exacerbations/6months & $2.0(2.75)$ & $0(1)$ & $<0.001^{\mathrm{b}}$ \\
PNL mg/d $n=12$ & $10(7.5)$ & $0(4.5)$ & $<0.005^{\mathrm{b}}$ \\
\hline
\end{tabular}

mean $\pm S D$, median (IQR), PNL prednisolone, ${ }^{a}$ paired t test, ${ }^{\mathrm{b}}$ Wilcoxon signed rank test

related to the number of radiofrequency treatments. The mean treatment in non-responders was $139 \pm 11$ activations, compared to $221 \pm 45$ activations in the responders, $p<0.01$.

\section{Radiofrequency treatment}

A mean of $211 \pm 50$ radiofrequency activations per patient (range: 121-305) were delivered. The relationship between activations delivered and treatment response measured by change in ACQ- 5 was explored by correlation and regression analysis. This is presented graphically in Fig. 1. The Pearson correlation coefficient for this relationship is $r=-0.43, p<0.05$. A P-P plot suggested that the data fitted most closely with a linear model. The regression line was given by the equation $A C Q$ delta $=0.92-0.01 x$ activations, $\mathrm{r}^{2}=0.18, p<0.05$. No correlation was observed between activations and change in $\mathrm{FEV}_{1} \%$ predicted $(r=-0.1, p=0.64)$.

\section{Technique}

Technique related differences were observed between proceduralists. Significantly fewer radiofrequency activations were delivered by proceduralist A $(155 \pm 24, n=8)$ by comparison with proceduralist $\mathrm{B}(235 \pm 24, n=10)$ or proceduralist C (244 $\pm 47, n=6)$ (ANOVA, $p<0.001)$. Proceduralist A's patient data were compared to those of

Table 2 Responder comparison

\begin{tabular}{llll}
\hline & Responders & Non-responders & $p$ \\
\hline $\mathrm{n}$ & 21 & 3 & - \\
Change in ACQ-5 & $-2.2 \pm 1.0$ & $+1.1 \pm 1.0$ & - \\
RF activations & $221 \pm 45$ & $139 \pm 11.4$ & $<0.01^{\text {a }}$ \\
Age (yrs) & $56.7 \pm 12.7$ & $47.0 \pm 8.9$ & NS \\
Male gender & $33 \%$ & $33 \%$ & NS \\
BMl kg/m ${ }^{2}$ & $28.9 \pm 7.0$ & $28.1 \pm 4.5$ & NS \\
Baseline FEV $\%$ & $61.2 \pm 16.8$ & $65.5 \pm 8.2$ & NS \\
BD response\% & $13.3 \pm 13.0$ & $14.9 \pm 8.5$ & NS \\
Salbutamol puffs/day & $8(12)$ & $4(5)$ & NS \\
\hline
\end{tabular}

$B D$ bronchodilator response \% change in FEV1, NS not significant, ${ }^{a}$ Mann-Whitney $U$ 


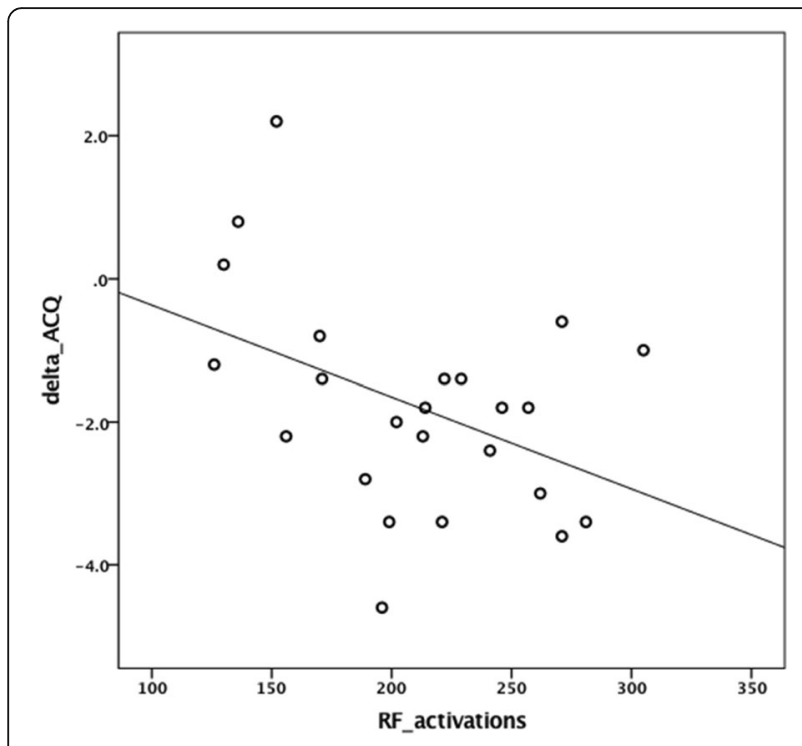

Fig. 1 Radiofrequency activations versus change in ACQ-5 after BT

Proceduralist $\mathrm{B}$ and $\mathrm{C}$ using an independent t-test, and the results are presented in Table 3. In an otherwise similar population of patients, the fewer activations by Proceduralist A may have reduced the magnitude of improvement in ACQ following BT.

A multivariate linear regression model was created to examine the contribution of variables that might influence the number of radiofrequency activations delivered to each patient. The variables examined included: age, gender, height, weight, baseline $\mathrm{FEV}_{1} \%$ predicted and proceduralist. The only variable found to significantly influence activations was the proceduralist, and the effect was strong, $r=0.727, p<0.001$.

\section{Adverse events}

In this series of 72 procedures, there were no deaths, and no readmissions for any cause within 30 days of a procedure. One case, on two occasions, required monitoring in Intensive Care immediately post procedure for

Table 3 Results comparison by proceduralist

\begin{tabular}{llll}
\hline & Proceduralist A & Proceduralist B $+C$ & $p$ \\
\hline $\mathrm{n}$ & 8 & 16 & \\
Activations & $155 \pm 24$ & $241 \pm 33$ & $<0.001^{\mathrm{a}}$ \\
Change in ACQ-5 & $-0.9 \pm 2.1$ & $-2.3 \pm 1.0$ & $<0.05^{\mathrm{a}}$ \\
Change in FEV $\%$ & $9.8 \pm 19.2$ & $6.1 \pm 15.1$ & $\mathrm{NS}$ \\
Age (yrs) & $47.8 \pm 10.7$ & $59.1 \pm 12.3$ & $<0.05^{\mathrm{a}}$ \\
Male gender & $50 \%$ & $25 \%$ & $\mathrm{NS}$ \\
BMl kg/m ${ }^{2}$ & $26.1 \pm 3.6$ & $29.9 \pm 7.8$ & $\mathrm{NS}$ \\
Baseline ACQ-5 & $3.0 \pm 1.0$ & $3.4 \pm 1.1$ & $\mathrm{NS}$ \\
Baseline FEV $\%$ & $61.3 \pm 9.0$ & $62.3 \pm 19.3$ & $\mathrm{NS}$ \\
\hline
\end{tabular}

${ }^{\text {andependent t-test }}$ an asthma exacerbation, and on one of these occasions received non-invasive ventilation before making a complete recovery. During the procedures, there were no instances of pneumothorax nor airway haemorrhage.

\section{Discussion}

The patients in this study comprise a group of severe asthmatics - more severe than were treated in the two larger randomized control trials of bronchial thermoplasty $[3,4]$. In the AIR and AIR2 trials, the mean prebronchodilator $\mathrm{FEV}_{1}$ was $72.7 \%$ and $77.8 \%$ predicted respectively, compared with $61.8 \%$ in the series we report. Furthermore, few patients were included in the above trials who required maintenance oral corticosteroids, monoclonal antibodies or immunosuppressants. However, the outcomes achieved across a broad range of parameters are superior to the AIR and AIR2 studies. Importantly, in this case series, a significant improvement in prebronchodilator $\mathrm{FEV}_{1}$ has been demonstrated following treatment - this being a missing feature of the larger clinical trials in BT. In this respect our series is similar to the RISA trial which also demonstrated a significant improvement in $\mathrm{FEV}_{1}$ with treatment. More severely obstructed patients it seems may have more to gain from BT.

This is the first study to have specifically examined the relationship between radiofrequency activations and clinical response. A significant relationship has been demonstrated between activations applied and improvement in ACQ-5. This is consistent with our understanding of the biological effect of BT on airway smooth muscle. However, two studies have examined the effect of BT on human airway smooth muscle obtained from endobronchial biopsies [7, 8]. Whilst both showed a reduction in airway smooth muscle with treatment, they were unable to correlate the degree of reduction with the number of activations applied. This difference from our study may well be explained by the wide variation observed between biopsies when measuring airway smooth muscle mass - this measurement error would serve to weaken any potential correlation with activations in a small study.

We believe that there is likely to be a minimum treatment level, below which therapy becomes less effective. The regression equation can be used to roughly approximate that in order to achieve an improvement in ACQ-5 of 0.5 units or greater, a target of 140 activations or more need to be delivered across the three treatment sessions. In this case series, $29 \%$ of activations occurred when the right lower lobe was treated, $28 \%$ when the left lower lobe was treated and $43 \%$ when the upper lobes were treated. Therefore, using the regression equation, this suggests that proceduralists should aim to deliver at least 40 activations to each of the lower lobes and 60 
activations to the combined upper lobes in order to achieve an ACQ change of 0.5 units. We acknowledge that this concept requires further validation in a larger study but the recommendations do appear to be consistent with previously published guidelines $[6,15]$. No correlation was observed between radiofrequency activations and change in $\mathrm{FEV}_{1}$, but the authors believe that this is because the magnitude of change in $\mathrm{FEV}_{1}$ with treatment is small $(+11 \%)$ by comparison with the magnitude of change in ACQ-5 (-55\%).

This study also demonstrates that, as in other areas of procedural medicine, there can be variation in technique amongst operators, and that this may affect patient outcomes. This variation only becomes apparent when comparative results are audited, as presented in Table 3. As a result of this observation, the videos taken during several procedures were reviewed and discussed by the proceduralists involved. It was concluded that the variation in activations between proceduralists was explained by variation in the distance the radiofrequency catheter was advanced into each subsegmental bronchus. The manufacturer recommends the catheter is advanced into the bronchial tree until the last depth marking on the catheter is just visible $[6,15]$ and a radiograph taken with the catheter at that position is shown in Fig. 2. However, it is possible to advance the catheter still further, into smaller airways until resistance is encountered, and this is demonstrated in the radiograph taken in Fig. 3. If this latter approach is adopted, this will result in a larger volume of subsegmental airways treated and higher number of activations. The authors believe that this is likely to be a common point of difference between proceduralists performing BT and may explain variation in outcomes between patients.

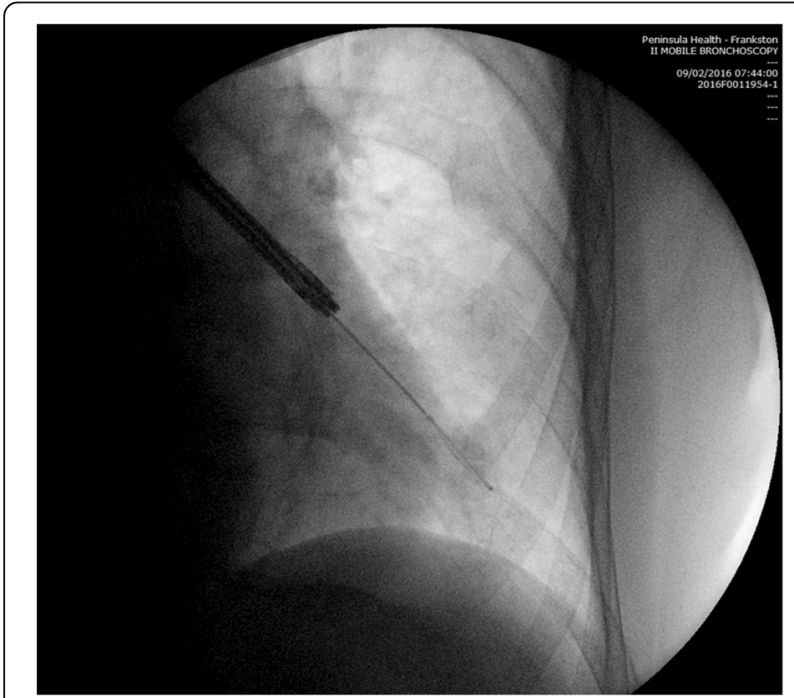

Fig. 2 RF catheter advanced to last visible depth marking

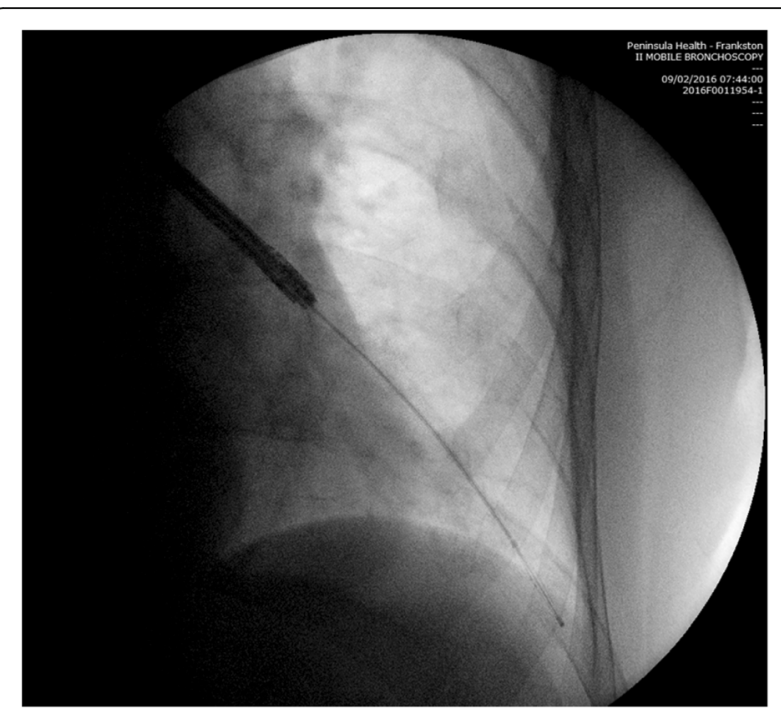

Fig. 3 RF catheter advanced distally until resistance encountered

Our study demonstrates that there is no loss of safety in performing BT with the catheter more peripherally advanced, and that the outcomes may be superior. However, this is a small study, and only $18 \%$ of the variation in change in ACQ-5 post-treatment is explained by variation in the number of activations applied. Therefore, there may be other factors, not yet identified which predict patient response to BT. This highlights the need for a future multivariate regression analysis of predictors of response to BT in a large cohort of patients. When completed, the Bronchial Thermoplasty Global Registry [16] may provide an ideal opportunity to undertake this analysis and further examine the role of radiofrequency activations.

\section{Conclusion}

This study demonstrates that there can be procedural differences between physicians in the application of radiofrequency treatment during BT, and that the resulting difference in activations can significantly affect patient outcomes.

\section{Abbreviations}

ACQ-5: Asthma control questionnaire-5 item version; ERS/ATS: European Respiratory Society/American Thoracic Society; FEV 1 : Forced expiratory volume in $1 \mathrm{~s}$; IQR: Interquartile range

\author{
Acknowledgements \\ Nil. \\ Funding \\ Nil.
}

Availability of data and materials

Please contact the primary author for data requests. 


\section{Authors' contributions}

DL had access to all study data and takes responsibility for data integrity and analysis. DL, Al and DF contributed patient data from their centres. DL, Al, DF and JS contributed to study design. All authors contributed to manuscript preparation. All authors read and approved the final manuscript.

\section{Ethics approval and consent to participate}

Approval to collate and audit data as part of quality assurance was provided by the Human Research and Ethics Committee at each participating institution.

\section{Consent for publication}

Not applicable.

\section{Competing interests}

The authors declare that they have no competing interests.

\section{Publisher's Note}

Springer Nature remains neutral with regard to jurisdictional claims in published maps and institutional affiliations.

\section{Author details}

'Department of Thoracic Medicine, Frankston Hospital, 2 Hastings Road, Frankston, VIC 3199, Australia. ${ }^{2}$ Faculty of Medicine, Nursing and Health Sciences, Monash University, Melbourne, VIC, Australia. ${ }^{3}$ Faculty of Medicine and Health Sciences, Macquarie University, Sydney, NSW, Australia. ${ }^{4}$ Department of Thoracic Medicine, Royal Brisbane and Women's Hospital, Herston, QLD, Australia. ${ }^{5}$ Eastern Health Clinical School, Box Hill Hospital and Monash University, Melbourne, VIC, Australia.

Received: 11 April 2017 Accepted: 29 June 2017

Published online: 04 July 2017

\section{References}

1. Chung KF, Wenzel SE, Brozek JL, et al. International ERS/ATS guidelines on definition, evaluation and treatment of severe asthma. Eur Respir J. 2014; 43(2):343-73.

2. Bousquet J, Mantzouranis E, Cruz AA, et al. Uniform definition of asthma severity, control, and exacerbations: document presented for the World Health Organization consultation on severe asthma. J Allergy Clin Immunol. 2010;126(5):926-38.

3. Cox G, Thomson NC, Rubin AS, et al. Asthma control during the year after bronchial thermoplasty. N Engl J Med. 2007;356(13):1327-37.

4. Castro M, Rubin AS, Laviolette $M$, et al. Effectiveness and safety of bronchial thermoplasty in the treatment of severe asthma: a multicenter, randomized, double-blind, sham-controlled clinical trial. Am J Respir Crit Care Med. 2010; 181(2):116-24

5. Pavord ID, Cox G, Thomson NC, et al. Safety and efficacy of bronchial thermoplasty in symptomatic, severe asthma. Am J Respir Crit Care Med. 2007:176(12):1185-91.

6. Mayse ML, Laviolette M, Rubin AS, et al. Clinical pearls for bronchial thermoplasty. J Bronchol. 2007;14(2):115-23.

7. Pretolani M, Dombret MC, Thabut G, et al. Reduction of airway smooth muscle mass by bronchial thermoplasty in patients with severe asthma. Am J Respir Crit Care Med. 2014;190(12):1452-4.

8. Chakir J, Haj-Salem I, Gras D, et al. Effects of bronchial Thermoplasty on airway smooth muscle and collagen deposition in asthma. Ann Am Thorac Soc. 2015;12(11):1612-8.

9. Miller JD, Cox G, Vincic L, Lombard CM, Loomas BE, Danek CJ. A prospective feasibility study of bronchial thermoplasty in the human airway. Chest. 2005; 127(6):1999-2006.

10. Keglowich LF, Borger $P$. The three A's in asthma - airway smooth muscle, airway remodeling \& angiogenesis. Open Respir Med J. 2015;9:70-80.

11. Dombret MC, Alagha K, Philippe Boulet $L$, et al. Bronchial thermoplasty: a new therapeutic option for the treatment of severe, uncontrolled asthma in adults. Eur Respir Rev. 2014;23(134):510-8.

12. Juniper EF, Svensson K, Mörk AC, Ståhl E. Measurement properties and interpretation of three shortened versions of the asthma control questionnaire. Respir Med. 2005;99(5):553-8.

13. Miller MR, Crapo R, Hankinson J, et al. General considerations for lung function testing. Eur Respir J. 2005;26(1):153-61.
14. Quanjer PH, Tammeling GJ, Cotes JE, Pedersen OF, Peslin R, Yernault JC. Lung volumes and forced ventilatory flows. Report working party standardization of lung function tests, European community for steel and coal. Official statement of the European respiratory society. Eur Respir J Suppl. 1993;16:5-40.

15. Wahidi MM, Kraft M. Bronchial thermoplasty for severe asthma. Am J Respir Crit Care Med. 2012;185(7):709-14.

16. Boston Scientific Corporation. Bronchial Thermoplasty global registry. In: ClinicalTrials.Gov [internet]. Bethesda: National Library of Medicine (US). 2000-8 April 2017. Available from: http://clinicaltrials.gov/show/NCT 02104856

\section{Submit your next manuscript to BioMed Central and we will help you at every step:}

- We accept pre-submission inquiries

- Our selector tool helps you to find the most relevant journal

- We provide round the clock customer support

- Convenient online submission

- Thorough peer review

- Inclusion in PubMed and all major indexing services

- Maximum visibility for your research

Submit your manuscript at www.biomedcentral.com/submit 